\title{
The effect of diet, lifestyle and/or cognitive interventions in Mild Cognitive Impairment: a systematic review
}

\author{
A.M McGrattan ${ }^{1}$, C.T McEvoy ${ }^{1}$, B. McGuinness ${ }^{1}$, M.C. McKinley ${ }^{1}$ and J.V. Woodside ${ }^{1}$ \\ ${ }^{1}$ Centre for Public Health, Queens University, Belfast, BT12 6BJ.
}

Mild Cognitive Impairment (MCI) is described as a transitional stage between the expected decline of normal ageing and that of dementia and is suggested to be the optimum stage for preventative intervention. The role of nutrition in the prevention of cognitive decline has been examined in terms of a range of nutrients/dietary patterns, investigating single nutrients, such as n-3 PUFA as well as whole diet interventions, such as the DASH diet, a ketogenic diet or the Mediterranean diet. A systematic review of Randomised Controlled Trials was conducted to examine the effect of diet, either alone or in combination with lifestyle and/or cognitive strategies, on cognitive health outcomes in patients with MCI. The search generated a total of 2130 articles and following the removal of duplicates and screening process, 12 studies remained and were included in the review.

\begin{tabular}{|c|c|c|}
\hline Reference & Intervention & Cognitive outcome measured \\
\hline Ma $(2016)^{(1)}$ & Folic Acid $(400 \mu \mathrm{g})(\mathrm{n}=180,6$ months $)$ & Memory*; Visuospatial skills* \\
\hline De Jager $(2012)^{(2)}$ & $\begin{array}{l}0.8 \mathrm{mg} \text { folic acid, } 0.5 \mathrm{mg} \text { vitamin } \mathrm{B} 12,20 \mathrm{mg} \text { vitamin } \mathrm{B} 6 \\
(\mathrm{n}=266,2 \text { years })\end{array}$ & Memory*; Executive function*; Global cognition \\
\hline Dekosky $(2008)^{(3)}$ & Gingko Biloba $(120-\mathrm{mg})(\mathrm{n}=482,6 \cdot 1$ years $)$ & Diagnosis of Dementia \\
\hline Lee $(2012)^{(4)}$ & $\begin{array}{l}\mathrm{n}-3 \text { fatty acids ( } 430 \mathrm{mg} \text { of DHA and } 150 \mathrm{mg} \text { of EPA })(\mathrm{n}= \\
36,12 \text { months) }\end{array}$ & $\begin{array}{l}\text { Memory*; Executive Function; Attention; Visuospatial Skills; Psychomotor speed; } \\
\text { Global cognitive function }\end{array}$ \\
\hline Petersen $(2005)^{(5)}$ & Vitamin E (2000 IU) $(n=769,3$ years $)$ & $\begin{array}{l}\text { Development Alzheimer's disease; Memory; Executive Function; Language; } \\
\text { Visuospatial skills; Overall Cognitive Function }\end{array}$ \\
\hline Krikorian $(2010)^{(6)}$ & Chromium Picolinate $(1000 \mathrm{mcg})(\mathrm{n}=26,12$ weeks $)$ & Memory; fMRI* \\
\hline Desideri $(2012)^{(7)}$ & $\begin{array}{l}\text { Cocoa Flavanols }(990 \mathrm{mg} / 520 \mathrm{mg} / 45 \mathrm{mg})(\mathrm{n}=90,8 \\
\text { weeks) }\end{array}$ & MMSE; Trail making test, Part A and B*; Verbal fluency test* \\
\hline Krikorian $(2010)^{(8)}$ & Concord grape juice $(\mathrm{n}=12,12$ weeks) & Memory* (verbal learning only) \\
\hline Krikorian $(2010)^{(9)}$ & Wild blueberry juice $(\mathrm{n}=9,12$ weeks) & Memory* (V-PAL test only) \\
\hline Horie $(2016)^{(10)}$ & $\begin{array}{l}\text { Nutritional counselling (healthy eating/calorie restriction) } \\
(\mathrm{n}=80,12 \text { months) }\end{array}$ & Memory; Executive Function; Language; Psychomotor Speed \\
\hline $\begin{array}{l}\text { Bayer-Carter } \\
(2011)^{(11)}\end{array}$ & $\begin{array}{l}\text { High-saturated fat } / \text { high-GI Vs low-saturated fat/low-GI } \\
\text { diet ( } \mathrm{n}=49,4 \text { weeks) }\end{array}$ & Memory; Executive Function; Motor Speed; AD Biomarkers* (CSF A $\beta 42$ only) \\
\hline Krikorian (2012) ${ }^{(12)}$ & $\begin{array}{l}\text { High carbohydrate Vs very low carbohydrate diet }(n=23 \text {, } \\
6 \text { weeks) }\end{array}$ & Memory \\
\hline
\end{tabular}

* indicates statistically significant difference between intervention and control/placebo at study completion $(\mathrm{p}<0 \cdot 05)$

Due to the heterogeneity across the studies, in terms of the dietary interventions and cognitive outcome measures used, results suggest that there is currently insufficient data to support the effect of diet on cognition in MCI patients. Therefore, there is a need for more robust RCTs to be conducted to explore the potential for dietary intervention to improve cognitive outcomes within this patient group.

1. Ma F, Wu T, Zhao J et al. (2016) J Gerontol A Biol Sci Med Sci, 1, 1376-1383.

2. de Jager CA, Oulhaj A, Jacoby R et al. (2012) Int J Geriatr Psychiatry, 27, 592-600.

3. DeKosky ST, Williamson JD, Fitzpatrick AL et al. (2008) JAMA, 300, 2253-2262.

4. Lee LK, Shahar S, Chin AV et al. (2013) Psychopharmacology, 225, 605-612.

5. Petersen RC, Thomas RG, Grundman M et al. (2005) N Engl J Med, 352, 2379-2388.

6. Krikorian R, Eliassen JC, Boespflug EL et al. (2010) Nutr. Neurosci, 13, 116-122.

7. Desideri G, Kwik-Uribe C, Grassi D et al. (2012) Hypertension, 60, 794-801.

8. Krikorian R, Nash TA, Shidler MD et al. (2010) Br J Nutr, 103, 730-734.

9. Krikorian R, Shidler MD, Nash TA et al. (2010) J Agric Food Chem, 58, 3996-4000

10. Horie NC, Serrao VT, Simon SS et al. (2016) J Clin Endocrinol Metab, 101, 1104-1112.

11. Bayer-Carter JL, Green PS, Montine TJ et al. (2011) Arch Neurol, 68, 743-752.

12. Krikorian R, Shidler MD, Dangelo K et al. (2012), Neurobiol Aging, 33, 425.e19-425.e27. 